\title{
Big Data und was nun? Neue Datenbestände und ihre Auswirkungen
}

\section{Vorwort der Herausgeber}

\author{
Ralf Thomas Münnich • Markus Zwick
}

(C) Springer-Verlag Berlin Heidelberg 2016

Mit der digitalen Revolution und der allgegenwertigen Verfügbarkeit des Internets und die Verbreitung von unter anderem Smartphones und Tablets sowie durch letztere hervorgerufene Änderungen des Nutzerverhaltens der Bevölkerung haben Forschern, Institutionen und Unternehmen der Privatwirtschaft mit Big Data ein neues Thema und gleichsam Big Challenges beschert. Sicherlich kann man von einem Hype sprechen und einige Versprechen von Big Data haben auch schon zu größeren Enttäuschungen geführt. Der Hype wird vergehen, aber die heute noch neuen digitalen Datenquellen werden bleiben.

An die Situation, dass die Computer immer schneller und leistungsfähiger werden, hat man sich mittlerweile gewöhnt. Was uns heute in der Statistik bewegt ist nicht unbedingt die weitere Entwicklung der Informationstechnik, sondern vor allem die Veränderung und Entwicklung der Information an sich. Informationen werden nicht länger nur primär produziert, sondern fallen in kaum bezifferbarer Menge, häufig als Nebenprodukt an. Aus dieser Sicht gefällt die angelsächsische Bezeichnung der neuen digitalen Datenquellen als organic data. Dies ist eine, aus hiesiger Sicht aber durchaus treffende Beschreibung der neuen digitalen Datenquellen, die Umgangssprachlich gerne als Big Data bezeichnet werden. Eine erweiterte Betrachtungsweise der organic data kann dabei auch administrative Daten umfassen (Shlomo et al. 2015).

R. T. Münnich $(\bowtie)$

Wirtschafts- und Sozialstatistik, Fachbereich IV, VWL, Universität Trier, Trier, Deutschland

E-Mail: muennich@uni-trier.de

\section{Zwick}

Institut für Forschung und Entwicklung in der Bundesstatistik, Statistisches Bundesamt, Wiesbaden, Deutschland

E-Mail: markus.zwick@destatis.de 
Die Kunst des Statistikers ist es, aus Masseninformationen entscheidungsrelevantes Wissen zu generieren. Bis dato war es so, dass Daten beziehungsweise Informationen durch den Statistiker meist zuerst produziert wurden. Dies hat sich mit den neunen digitalen Datenquellen verändert. Diese liegen vor und es wird mehr und mehr zur Aufgabe des Statistikers, diese zur adäquaten Beschreibung realer Begebenheiten in Wirtschaft und Gesellschaft zu verwenden. Dies bedeutet nicht, dass organic data Erhebungsdaten ersetzen werden, zumindest nicht in der kurzen bis mittleren Sicht. Innerhalb von Multi-Source-Anwendungen werden neue digitale Datenquellen in einem ersten Schritt bestehende und bewährte Verfahren in der Statistik ergänzen und erweitern.

Auch dies wird aber nicht in der kurzen Frist geschehen können. Viele Fragen zu diesen neuen digitalen Datenquellen müssen noch geklärt werden, bevor sie $a d$ äquat eingesetzt werden können, wie z. B. innerhalb der amtlichen Statistik, bei der Politikberatung oder gar vor Gericht. Im Vordergrund stehen sicher Fragen zur Qualität, zum Datenschutz oder der dauerhaften Verfügbarkeit, um nur einige Herausforderungen an dieser Stelle zu nennen. Trotz der vielen Aufgaben, die noch vor einem breiteren Einsatz der neuen digitalen Datenquellen zu erledigen sind, ist es notwendig mit diesem Prozess zu beginnen - sinnvollerweise als interdisziplinäre Forschung beziehungsweise Anwendung zwischen amtlicher und methodischer Statistik auf der einen Seite aber auch Informatik und (numerischer) Mathematik beziehungsweise Optimierung auf der anderen Seite.

Was genau wir in diesem Zusammenhang unter Big Data zu verstehen haben, welche Vorzüge und Nachteile Big Data bringen und welche Anforderungen an die Statistik aus diesem Themenkomplex erwachsen, all das soll in diesem Sonderband von AStA Wirtschafts- und Sozialstatistisches Archiv im Rahmen von acht spannenden Beiträgen aufgezeigt werden. Was Sie, liebe Leserinnen und Leser, im Detail erwartet, erfahren Sie im Folgenden.

Sobald das Thema Big Data diskutiert wird, fällt über kurz oder lang auch der Name Twitter. Der Kurznachrichtendienst versorgt seit 2006 seine Nutzer, follower genannt, mit allen erdenklichen auf 140 Zeichen komprimierten Informationen, seien sie banal wie die tweets von Prominenten (die Popsängerin Katy Perry hat derzeit laut Medienberichten über 90 Mio. follower) oder bedeutsam wie die Kommunikation zwischen ägyptischen Revolutionären im Rahmen der Demonstrationen auf dem Tahrir-Platz in Kairo 2011. Bei, laut Eigenangaben des Unternehmens aus dem März 2016, 305 Mio. aktiven Nutzern weltweit, eröffnet sich mit Twitter eine weitere reiche Datenquelle (s. ZEIT ONLINE 2016). Dass Twitter-Daten auch in sozioökonomischen Studien Verwendung finden können, zeigen Marchetti et al. (2016) in ihrem Beitrag. Sie nutzen einen aus Twitter-Daten konstruierten Emotions-Indikator als Hilfsvariable in einem Small Area-Modell mithilfe dessen sie den Anteil der Nahrungsmittelausgaben an den gesamten Konsumausgaben italienischer Haushalte auf Provinzebene schätzen. Die Verwendung der Kurznachrichtendienst-Daten führt zu besseren Prädiktionen und ermöglicht eine präzisere Schätzung als in einem Modell ohne diese Daten. Nicht unerwähnt bleiben im Beitrag aber auch Probleme, die durch Selbstselektion und Messfehler induziert werden.

Mit der Forstwirtschaft nutzt ein im allgemeinen Bewusstsein eher noch als ana$\log$ angesehener Wirtschaftszweig seit längerer Zeit bereits Datenmaterial, das mitt- 
lerweile als Big Data bezeichnet werden kann. Wie Münnich et al. (2016) zeigen, helfen mit flugzeug- und (vor allem) satellitengestützten Sensorsystemen gewonnene Fernerkundungsdaten bei der für die forstliche Planung unabdingbaren Forstinventur. Für letztere müssen Bestandsschätzungen herangezogen werden. Diese sollten wiederum idealerweise auch auf sehr kleinen Gebietseinheiten möglichst präzise sein. Die Autoren stellen in ihrem Beitrag einerseits aktuell diskutierte Verfahren zur Schätzung unter Nutzung von Hilfsinformationen vor und führen andererseits konkrete Schätzungen für Holzvorräte in Rheinland-Pfalz durch. Offenkundig muss allerdings auch die Güte und Beschaffenheit von Vor- und Zusatzinformationen, die in Modellen genutzt werden sollen, diskutiert werden. Zudem führt bei der Verwendung mehrerer Hilfsvariablen der Fluch der Dimensionalität (siehe Bellman 1961) zu enormem Speicher- und Rechenaufwand. Speziell dem letztgenannten Punkt im Zusammenhang mit nichtlinearen Modellierungen widmet sich der Beitrag.

Bei einem so umfassenden Phänomen wie dem Thema dieses Sonderbands ist auch ein Blick weg von Spezifika und hin zu eher philosophischen Überlegungen gerechtfertigt.

Einigen Beobachtern ist bereits die teils inflationäre Nutzung des Begriffs Big Data ein Graus und manch einer bezweifelt, dass sich Big Data lange halten wird und glaubt eher, dass es eine Art kurzfristige Modeerscheinung ist. Auf der anderen Seite stehen viele Datennutzer, die erst jetzt wirklich erfassen, wie die Datenflut konstruktiv genutzt werden kann. Für sie ist Big Data gekommen um zu bleiben. Askitas (2016) sieht zwar eine Ende von Big Data kommen, allerdings nur, da mittelfristig sämtliche Daten Big Data sein werden, wie er argumentiert. Der Autor beschäftigt sich anhand einiger Beispiele mit der unbedingt zulässigen Frage, wieviele Daten wir tatsächlich brauchen und nutzen können. Kann nicht in manchen Fällen ein Zuviel an Daten ebenso schädlich sein wie ein Zuwenig, je nachdem um welche konkrete Anwendung, welchen konkreten Nutzer es geht? Ebenso spannend ist ein Blick auf die Auswirkungen des massiven Datensammelns und Datenmaterials auf das Verhalten der Menschen an sich. Kann es sein, dass die Beschäftigung mit Big Data bereits unser Verhalten, und damit letztlich auch die gewonnenen Daten selbst, ändert? Der Autor gibt dem Leser hier einige Denkanstöße.

Neue Berufsbezeichnung wie Data Scientists oder iStatistician deuten auf den Wandel in den Anforderungsprofilen von ausgebildeten Empirikern hin. Data Scientists werden in der Forschung, in Organisationen und in der Privatwirtschaft händeringend gesucht um der steigenden Datenflut Herr zu werden und aus ihr wertvolle Informationen und Schlussfolgerungen zu ziehen (siehe Sommer 2013). Neue Berufsfelder im Spannungsfeld zwischen Statistik, Mathematik und Informatik entstehen. Zwick (2016) geht in seinem Beitrag darauf ein, inwieweit die statistische Ausbildung weiterentwickelt werden sollte, um den deutlich gestiegenen und weiter steigenden Bedarf an empirisch ausgebildeten Experten bedienen zu können. Dies betrifft zum einen die universitäre Ausbildung in Bachelor- wie Masterprogrammen, hier ist der European Master in Official Statistics (EMOS) eine erste Antwort. Zum anderen ist aber auch die Weiterbildung von Forschern und Statistikern in den Institutionen anzupassen. Hier sind die Stichworte life-long-learning aber auch statistical literacy, da auch die Nutzer von empirischen Informationen ein immer größeres statistisches Verständnis zur sachgerechten Nutzung der Informationsprodukte be- 
nötigen. Die Veränderung der statistischen Aus- und Weiterbildung ist hierbei nicht nur eine Frage der zu vermittelnden Methoden und Inhalte, sondern auch der didaktischen Möglichkeiten. E-Learning, Webinare, MOOCs sind hierzu die Stichworte.

Kauermann und Küchenhoff (2016) nähern sich in ihrem Beitrag dem nicht trennscharf zu definierenden Konstrukt Data Science im Kontext von Big Data an und grenzen es zunächst vom herkömmlichen Verständnis der Disziplinen Statistik und Informatik ab. Sehen sich die beiden Disziplinen tatsächlich einem gewissen Konkurrenzkampf ausgesetzt? Die Autoren kommen letztlich zu dem Schluss, dass der Statistik und dem statistischen Denken auch im Zeitalter von Big Data noch eine zentrale Rolle zukommt und somit das Science in Data Science nicht an Gewicht verlieren wird. Sicher ist in jedem Fall, dass sich neue und hochspannende interdisziplinäre Forschungsfragen auftun werden. Ebenso sicher ist aber auch, dass Data Science eine Herausforderung und Bereicherung für die statistische Aus- und Weiterbildung an Hochschulen, in Institutionen und in Unternehmen darstellt - wie der an der LMU München neu eingeführte interdisziplinäre Studiengang zeigt.

Big Data wird in starkem Maße auch die amtliche Statistik verändert. Neue digitale Datenbestände sind eine wertvolle Möglichkeit künftig gegebenenfalls amtliche Statistiken aktueller und ressourcen-schonender zu produzieren. Dies bedarf aber einer sehr strukturierten und detaillierten Herangehensweise der Statistischen Ämter. Der Beitrag von Wirthmann (2016) beschreibt hierzu das koordinierte Vorgehen der Statistischen Ämter innerhalb des Europäischen Statistischen Systems (ESS). Das Scheveningen-Memorandum der Leiterinnen und Leiter der Statistischen Ämter des ESS im Jahre 2013 war der Beginn einer intensiven Zusammenarbeit der europäischen Statistischen Ämter zum Thema Big Data. Der Beitrag geht beginnend auf diese Historie ein und beschreibt die gegenwärtig laufenden Projekte im Rahmen der ESS Big Data Action Plan and Roadmap 1.0 sowie die nächsten vorgesehen Schritte zur Integration der neunen digitalen Daten in amtliche Statistiken.

Nachdem Indikatoren zur Beschreibung gesellschaftlicher und wirtschaftlicher Phänomene, in den achtziger und neunziger Jahren des letzten Jahrhunderts, eine größere Bedeutung, insbesondere in den Sozialwissenschaften, hatten, sind sie nun, nach zwei Jahrzenten geringerer Relevanz, als Basis politischer Entscheidungsfindung wieder hochaktuell. Schnorr-Bäcker (2016) diskutiert in ihrem Beitrag die Rolle von Indikatoren als Politik begleitendes statistisches Monitoring und welche Bedeutung künftig gegebenenfalls neue digitale Datenquellen zur Entwicklung von Indikatoren in verschiedensten Politikbereichen annehmen können. Hervorzuheben sind hier die auf der UN Ebene entwickelten Sustainable Development Goals und das hierzu umfangreich zu generierende Indikatorensystem, welches mit bestehenden amtlichen Daten nur bedingt zu bedienen ist. Der Beitrag erläutert für verschiedene Beispiele, wie neue digitale Datenquellen, wie beispielsweise Mobilfunkdaten, Satellitenaufnahmen, Crowd Scoring oder aber auch Daten aus sozialen Medien zur Entwicklung von Indikatoren künftig genutzt werden können.

Einen sehr praktischen Blick auf Big Data und die Verwendung dieser neuen digitalen Datenquellen für amtliche Regionalstatistiken gibt Eichhorn (2016) in seinem Beitrag. Nach der Darlegung der gegenwärtigen Verfahren und Entwicklungstendenzen in den Regionalstatistiken, die in starkem Maße in Deutschland durch die Statistischen Ämter der Länder und seitens der Kommunen betrieben wird, erfolgt 
ein kritischer Blick hinsichtlich der Möglichkeiten zur Integration alternativer digitaler Datenbestände im Bereich dieser amtlichen Statistiken. Wesentliche Aspekte, wie die dauerhafte Verfügbarkeit der Daten, Datenschutz- und Qualitätsaspekte werden dabei angesprochen. Als Schlussfolgerung beschreibt der Beitrag die Notwendigkeit der sehr ausführlichen Prüfung neuer digitaler Datenquellen, vor einen Einsatz dieser Daten, dies für amtliche Statistiken allgemein und für die Regionalstatistiken im Speziellen.

Und nun wünschen wir Ihnen, liebe Leserinnen und Leser, viel Spaß bei der Lektüre dieses Sonderbands von AStA Wirtschafts- und Sozialstatistisches Archiv.

\section{Literatur}

Askitas N (2016) Big Data is a Big Deal but how much data do we need? Wirtsch Sozialstat Arch. doi:10. 1007/s11943-016-0191-3

Bellman R (1961) Adaptive control processes: a guided tour. Princeton University Press, Princeton

Eichhorn L (2016) Big Data in der Regionalstatistik. Wirtsch Sozialstat Arch. doi:10.1007/s11943-0160187-z

Kauermann G, Küchenhoff H (2016) Statistik, Data Science und Big Data. Wirtsch Sozialstat Arch. doi:10. 1007/s11943-016-0188-y

Marchetti S, Giusti C, Pratesi M (2016) The use of twitter data to improve small area estimates of households' share of food consumption expenditure in Italy. Wirtsch Sozialstat Arch. doi:10.1007/s11943016-0190-4

Münnich R, Wagner J, Hill J, Stoffels J, Buddenbaum H, Udelhoven T (2016) Schätzung von Holzvorräten unter Verwendung von Fernerkundungsdaten. Wirtsch Sozialstat Arch. doi:10.1007/s11943-0160186-0

Schnorr-Bäcker S (2016) Politik begleitendes statistisches Monitoring und neue Datenquellen. Wirtsch Sozialstat Arch. doi:10.1007/s11943-016-0192-2

Shlomo N, Goldstein H (2015) Editorial: Big data in social research. J R Stat Soc Ser A 178(Part 4):787-790

Sommer S (2013) Data Scientists - Schatzsucher im Datenmüll. Frankfurter Allgemeine Zeitung. http:// www.faz.net/aktuell/beruf-chance/data-scientists-schatzsucher-im-datenmuell-12653635.html. Zugegriffen: 28. Jul 2016

Wirthmann A (2016) Big Data im Europäischen Statistischen System - Beitrag zur Reaktion des Europäischen Statistischen Systems auf die Big Data Herausforderung. Wirtsch Sozialstat Arch. doi:10. 1007/s11943-016-0195-z

ZEIT ONLINE (2016) Twitter nennt erstmals Nutzerzahlen für Deutschland. http://www.zeit.de/digital/ 2016-03/soziale-medien-twitter-nutzerzahlen-deutschland. Zugegriffen: 28. Jul 2016

Zwick M (2016) Statistikausbildung in Zeiten von Big Data. Wirtsch Sozialstat Arch. doi:10.1007/s11943016-0185-1 